\title{
Suppression of defect propagation in semiconductors by pseudomorphic layers
}

\author{
Y. C. Chen, J. Singh, and P. K. Bhattacharya \\ Solid State Electronics Laboratory, Department of Electrical Engineering and Computer Science, \\ The University of Michigan, Ann Arbor, Michigan 48109-2122
}

(Received 17 March 1993; accepted for publication 25 May 1993)

\begin{abstract}
The propagation of defects in semiconductor heterostructures has been studied both theoretically and experimentally. The simple model shows that defects originating from lattice-matched regions can be prevented from entering, or can be trapped by, a pseudomorphic layer, depending on the signs of the strain induced by the defect and the strain in the pseudomorphic layer. A pseudomorphic layer can therefore prevent the defect from propagating across in and entering the critical active region of a device. Experimentally, the photoluminescence intensities of $\mathrm{Al}_{0.4} \mathrm{Ga}_{0.6} \mathrm{As} / \mathrm{GaAs}$ quantum wells with and without pseudomorphic $\operatorname{In}_{0.2} \mathrm{Ga}_{0.8}$ As layers for prevention of defect propagation have been compared. GaAs substrates of high etch pit density were used to generate defects below the quantum well and silicon implantation was used to generate defects above it. The elevated temperatures during molecular-beam-epitaxial growth and postimplant rapid thermal annealing serve to assist in defect propagation in the respective regions of the heterostructure. The structures with pseudomorphic $\mathrm{In}_{0.2} \mathrm{Ga}_{0.8}$ As layers consistently showed much higher quantum-well photoluminescence intensities than those without the pseudomorphic layers. These results indicate smaller defect densities in the quantum wells with pseudomorphic layers and strongly support the defect propagation model.
\end{abstract}

\section{INTRODUCTION}

In recent years, strained layers have been widely used in semiconductor devices to alter the band structure of materials in the active regions. This has led to the development of novel and high-performance electronic and optoelectronic devices. ${ }^{1-5}$ Strained quantum-well lasers, in particular, have provided the lowest threshold lasing due to reduction in the hole density of states due to biaxial strain. ${ }^{3-5}$ An important issue in such pseudomorphic devices is that of structural stability and device reliability.

As long as the pseudomorphic layer is below its critical thickness, in principle the structure has a lower free energy than the structure produced if dislocations were to arise. However, the strained structure has a higher energy than a lattice-matched unstrained structure. As a result the excess energy needed to generate dislocations is lower in the pseudomorphic layer. This may suggest that pseudomorphic layers may be less stable and the devices made are less reliable. However, this has not been observed and, on the contrary, there is evidence that strained lasers may be even more reliable and have a longer lifetime than unstrained lasers. ${ }^{6,7}$ Another factor that may affect the structural stability and device reliability is defect propagation. Since reliability in devices is an extremely important aspect of device technology, it is important to examine the problem of defect propagation.

In general, a large class of complicated structural defects, e.g., point defects and dislocations, can arise in a device structure as a result of growth, operation, handling, etc. It has been observed that, ${ }^{8}$ in III-V semiconductors, dislocations prefer to propagate along the $\{111\}$ planes. Therefore, on (100)-oriented substrates, dislocations orig- inating from the substrate or highly mismatched interfaces would prefer to propagate through the epitaxial layer toward the surface. Due to advanced growth and preparation techniques, substrates with very small dislocation densities are now available. Therefore, unless one grows latticemismatched layers with thickness larger than the critical thickness, the major defects in the growing layer should be point defects such as impurities and lattice vacancies. In this article we consider the propagation of point defects, which usually proceeds by a diffusion process. A simple model is presented in Sec. II to compare the energy required for a defect to propagate from a lattice-matched region into a pseudomorphic region with the energy required for a defect to propagate only inside a latticematched region. In Sec. III we describe the experimental techniques and samples used to verify our model. The experimental results are presented and discussed in Sec. IV, and the results are summarized in Sec. V.

\section{THEORY}

We examine here a simple source of defect generation in pseudomorphic layers. This process involves a defect outside the pseudomorphic layer in the lattice-matched region gradually penetrating into the pseudomorphic layer. This defect may have its origin in the substrate. The question we will address is whether this external defect will propagate into the pseudomorphic region or it will prefer to propagate through the lattice-matched region only. The two situations are shown schematically in Fig. 1(a). Defect propagation will involve straining the chemical bonds to distort them and thus introduce local strain. This is shown schematically in Fig. 1(b) where a vacancy is 


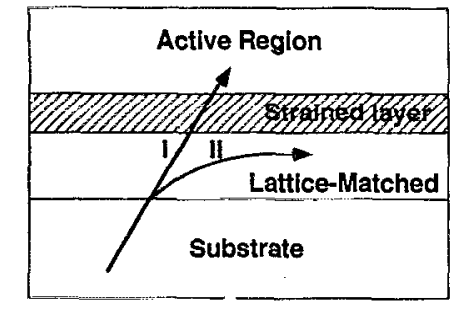

(a)

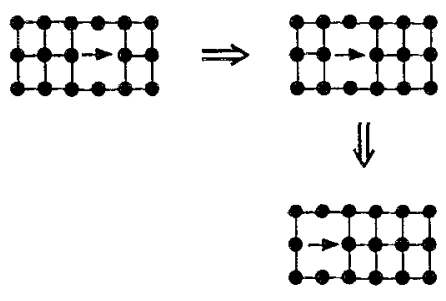

(b)

FIG. 1. (a) Schematic illustration of defect propagation in a mismatched semiconductor heterostructure. The defect might propagate through the lattice-matched region only (path II) or might propagate into the pseudomorphic region (path $I$ ); (b) the propagation of a lattice vacancy.

shown to progress through the crystal. The defect will prefer to follow a path where the force required to start the distortion, $-\partial E / \partial \epsilon$ ( $\epsilon$ being the strain) is the least. The quantity $E$ represents the total energy associated with, say, the unit-cell volume near the bond. Let us consider the strain energy in a pseudomorphic film grown on (001)oriented substrate. The strain tensor is

$$
\begin{aligned}
& \epsilon_{x x}=\epsilon_{y y}=\epsilon_{\|}=\epsilon=\frac{a_{s}-a_{0}}{a_{s}}, \\
& \epsilon_{z x}=\frac{-2 c_{12}}{c_{11}} \epsilon,
\end{aligned}
$$

where $a_{s}$ and $a_{0}$ are the substrate and epilayer lattice constants and $c_{11}$ and $c_{12}$ are the elastic constants. Note that, in this definition, $\epsilon<0$ when the epilayer is compressively strained. The strain is confined to the pseudomorphic layer alone and does not extend into the lattice-matched regions. The strain energy per unit cell of a film is ${ }^{9}$

$$
\begin{aligned}
E= & a_{0}^{3}\left[\frac{1}{2} c_{11}\left(\epsilon_{x x}^{2}+\epsilon_{y y}^{2}+\epsilon_{z z}^{2}\right)+c_{12}\left(\epsilon_{x x} \epsilon_{y y}+\epsilon_{y y} \epsilon_{z z}+\epsilon_{z z} \epsilon_{x x}\right)\right. \\
& \left.+\frac{1}{2} c_{44}\left(\epsilon_{x y}^{2}+\epsilon_{y z}^{2}+\epsilon_{z x}^{2}\right)\right] .
\end{aligned}
$$

Substituting for the strain tensor, we have

$$
E=\epsilon^{2} a_{0}^{3}\left(c_{11}+c_{12}-\frac{2 c_{12}^{2}}{c_{11}}\right) .
$$

In the lattice-matched regions the strain energy is zero. Let us now consider the situation where a defect in the latticematched region tends to propagate in the epitaxial layer by creating a strain field of the kind discussed in the context of Fig. 1. The qualitative aspects of our results are not affected by the precise nature of the strain. If $\delta \epsilon_{x x}$ is the excess distortion produced (i.e., in excess of the equilibrium value) in the initially unstrained lattice-matched region (path II), the energy generated in a unit cell of volume $a_{0}^{3}$ is

$$
\delta E_{\mathrm{II}}=a_{0}^{3}\left(\frac{1}{2} c_{11} \delta \epsilon_{x x}^{2}\right) .
$$

The force needed to produce the distortion is now

$$
\frac{\partial \delta E_{\mathrm{II}}}{a_{0} \partial \delta \epsilon_{x x}}=a_{0}^{2} c_{11} \delta \epsilon_{x x},
$$

which is the usual restoring force encountered in lattice dynamics problems.

If the distortion is to propagate into the strained region as well, as shown in Fig. 1 (path I), the excess strain energy is

$$
\delta E_{\mathrm{I}}=a_{0}^{3}\left(c_{11} \epsilon_{x x} \delta \epsilon_{x x}+c_{12} \epsilon_{y y} \delta \epsilon_{x x}\right)+O\left(\delta \epsilon_{x x}^{2}\right) .
$$

The force required to produce the excess strain is now

$$
\frac{\delta E_{\mathrm{I}}}{a_{0} \delta \epsilon_{\mathrm{xx}}}=a_{0}^{2}\left(c_{11} \epsilon_{x x}+c_{12} \epsilon_{y y}\right) .
$$

In the initial stage of defect propagation in a pseudomorphic structure, the magnitude of $\delta \epsilon_{x x}$ is very small, compared to that of $\epsilon_{x x}$. Consequently, we have $\delta E_{\mathrm{I}}>\delta E_{\mathrm{II}}$ and $\left|\delta E_{\mathrm{I}} / \delta \epsilon_{x x}\right|>\left|\partial \delta E_{\mathrm{II}} / \partial \delta \epsilon_{x x}\right|$. If both $\epsilon_{x x}$ and $\delta \epsilon_{x x}$ are negative, i.e.,the strain induced by the defect and the strain in the pseudomorphic layer are compressive, it will be much easier for the defect-induced strain to proceed along path II of Fig. 1 (a), where $\epsilon_{x x}=\epsilon_{y y}=0$. The cost of introducing more strain in the compressively strained region is very high, the strained region will therefore tend to repel any defect of $\delta \epsilon_{x x}<0$ from entering it. Once the strain proceeds via the path II, the defects will "flow" away from the strained region. On the other hand, if the defect-induced strain is tensile, $\delta \epsilon_{x x}>0$ (e.g., a vacancy), the total energy minimum favors the defect to propagate into the compressively strained region. This is analogous to a compressed spring. It is difficult to compress the spring further, but it is much easier to stretch the spring back to equilibrium. Once a defect of $\delta \epsilon_{x x}>0$ propagates into the compressively strained region, it will be trapped since it requires more energy for the defect to return to the lattice-matched region than to stay in the strained layer. The previous discussions are for compressively strained layers. If the pseudomorphic layer is under tensile strain, a defect of $\delta \epsilon_{x x}>0$ will be repelled from entering the strained layer while a defect of $\delta \epsilon_{x x}<0$ will prefer to enter and stay in it. A pseudomorphic layer thus can prevent a defect from propagating across $i t$, regardless of the signs of the strains of the pseudomorphic layer and the defect.

\section{EXPERIMENTAL TECHNIQUES}

We have carried out a carefully designed experiment to verify our model. The idea involves creating defects outside a quantum well (QW) and allowing them to propagate. The quality of a $\mathrm{QW}$ bounded by pseudomorphic layers is then compared to that without the pseudomorphic layers. If the pseudomorphic layer can really block defect propa- 


\begin{tabular}{rc}
\hline $150 \AA$ & GaAs \\
\hline $2400 \AA$ & $\mathrm{Al}_{0.4} \mathrm{Ga}_{0.6} \mathrm{As}$ \\
\hline $100 \AA$ & $\mathrm{GaAs} \mathrm{Quantum}_{\mathrm{Well}}$ \\
\hline $700 \AA$ & $\mathrm{Al} 0.4 \mathrm{Ga}_{0.6} \mathrm{As}$ \\
\hline & GaAs Substrate
\end{tabular}

(a)

\begin{tabular}{cc}
\hline $150 \AA$ & GaAs \\
\hline $2000 \AA$ & $\mathrm{Al}_{0.4} \mathrm{Ga}_{0.6} \mathrm{As}$ \\
\hline $80 \AA$ & $\ln _{0.2} \mathrm{Ga}_{0.8} \mathrm{As}$ Guarding Layer \\
\hline $400 \AA$ & $\mathrm{Al}_{0.4} \mathrm{Ga}_{0.6} \mathrm{As}$ \\
\hline $100 \AA$ & $\mathrm{GaAs}_{\mathrm{Quantum}}$ Well \\
\hline $400 \AA$ & $\mathrm{Al}_{0.4} \mathrm{Ga}_{0.6} \mathrm{As}$ \\
\hline $80 \AA$ & $\ln _{0.2} \mathrm{Ga}_{0.8} \mathrm{As}_{\mathrm{Suarding}}$ Layer \\
\hline $300 \AA$ & $\mathrm{Al}_{0.4} \mathrm{Ga}_{0.6} \mathrm{As}$ \\
\hline \multicolumn{3}{c}{ GaAs Substrate }
\end{tabular}

(b)

FIG. 2. GaAs $/ \mathrm{Al}_{0.4} \mathrm{Ga}_{0.6}$ As quantum-well structures (a) without and (b) with strained $\mathrm{In}_{0.2} \mathrm{Ga}_{0.8} \mathrm{As}$ guarding layers.

gation, the bound QW should have a higher optical efficiency than the QW without the pseudomorphic layers. In our experiments the defects originate from the substrate or are introduced intentionally by implantation damage.

The experimental samples are GaAs-based heterostructures grown by molecular-beam epitaxy (MBE). Three sets of samples were used in the study, each consisting of two different structures. Structure A [Fig. 2(a)] consists of, from bottom to top, a $700 \AA \mathrm{Al}_{0.4} \mathrm{Ga}_{0.6}$ As layer, a $100 \AA \mathrm{GaAs} \mathrm{QW}$, a $2400 \AA \mathrm{Al}_{0.4} \mathrm{Ga}_{0.6}$ As layer, and finally a $150 \AA$ GaAs cap layer to prevent the top $\mathrm{Al}_{0.4} \mathrm{Ga}_{0.6} \mathrm{As}$ layer from being oxidized. Structure $\mathrm{B}$ [Fig. $2(\mathrm{~b})]$ is similar to structure $\mathrm{A}$ except that two additional $80 \AA \mathrm{In}_{0.2} \mathrm{Ga}_{0.8} \mathrm{As}$ layers were inserted in the $\mathrm{Al}_{0.4} \mathrm{Ga}_{0.6} \mathrm{As}$ layers, $400 \AA$ away from the $\mathrm{GaAs} \mathrm{QW}$ on each side. Semiinsulating GaAs substrates with etch-pit density (EPD) of $5 \times 10^{4} \mathrm{~cm}^{-2}$ were used. The growth temperature for $\mathrm{In}_{0.2} \mathrm{Ga}_{0.8} \mathrm{As}$ and $\mathrm{GaAs}$ were 520 and $600^{\circ} \mathrm{C}$, respectively. The $\mathrm{Al}_{0.4} \mathrm{Ga}_{0.6}$ As layers were grown at 670,640 , and $600{ }^{\circ} \mathrm{C}$ for sets 1,2 , and 3 , respectively. No GaAs smoothing buffer or superlattice was deposited before the growth of these structures. The intentional use of high-EPD substrates and the thin bottom $\mathrm{Al}_{0.4} \mathrm{Ga}_{0.6} \mathrm{As}$ layer is to observe defect propagation from the substrate, which may occur during MBE growth.

Damages were also introduced intentionally into the top $\mathrm{Al}_{0.4} \mathrm{Ga}_{0.6} \mathrm{As}$ layer of both structures by silicon implantation. The implant profile was designed such that $R+3 \Delta R \sim 0.19 \mu \mathrm{m}$, where $R$ is the projected range and $\Delta R$ is the standard deviation in the direction normal to the surface. Since the tail of the implantation profile is about $650 \AA$ away from the GaAs QW, charge transfer into the $\mathrm{QW}$ is a minimum. The implanted samples were then subjected to rapid thermal annealing (RTA) at $800^{\circ} \mathrm{C}$ for 15 $s$. The implantation resulted in significant changes in the

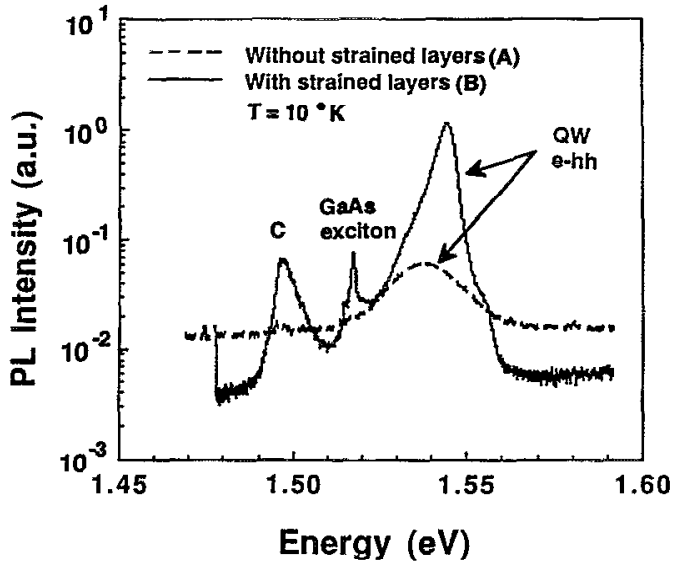

FIG. 3. Low-temperature photoluminescence spectra of as-grown $\mathrm{GaAs} / \mathrm{Al}_{0.4} \mathrm{Ga}_{0.6}$ As quantum wells with (solid curve) and without (dashed curve) strained $\mathrm{In}_{0.2} \mathrm{Ga}_{0.8}$ As guarding layers. Note that the intensity is in log scale.

structural properties of the samples, as observed from double-crystal $x$-ray rocking curve measurements. However, after RTA the samples showed x-ray rocking curves identical to that of as-grown samples. It should be noted that in addition to annealing of implantation damage, RTA can also assist in the propagation of residual damage. Photoluminescence (PL) measurements were made on the samples before and after the implantation-RTA treatment to compare the optical efficiency of the QWs in structures $A$ and $B$.

We are interested in the QW PL intensity ratios between structures $A$ and $B$ of each set and between the annealed and unannealed samples of each structure. To accurately measure the PL intensity ratio, we mounted the pair of samples to be compared together in the cryostat near the centers of the viewports. Measurements of each sample were made by vertically adjusting the cryostat position while maintaining the path of the incident laser beam fixed. The PL signal was maximized by fine adjustment of the focusing lenses. We have repeated the measurements for set 3 several times. Each time the samples were remounted and their positions were switched. In all the repetitions, we obtained the same value for the ratio of the maximized PL intensities of structures $\mathrm{A}$ and $\mathrm{B}$. Therefore, we believe that our measurement configuration and method do not introduce much uncertanty in the results and are suitable for the comparison in this study.

\section{RESULTS AND DISCUSSION}

Figure 3 shows the PL spectra of the as-grown samples of structures A and B from set 1. Besides the QW peak, in structure $B$ there are two additional peaks appearing at 1.517 and $1.496 \mathrm{eV}$. These are from the GaAs substrate and possibly originate from bound excitons and carbon impurities, respectively. As can be seen from this figure, structure B (with strained layers) shows much stronger QW PL intensity than structure A (without strained layers). The PL intensity ratio of the $Q W$ peaks for the two 


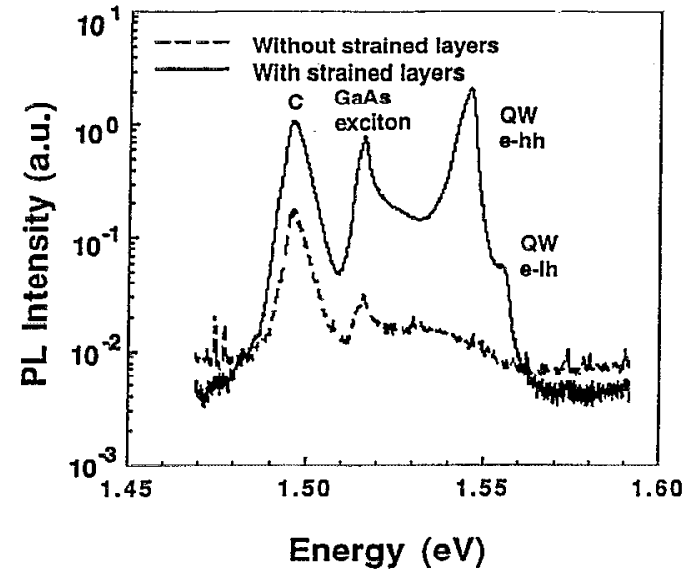

FIG. 4. Low-temperature photoluminescence spectra measured in implantation-RTA-treated $\mathrm{GaAs} / \mathrm{Al}_{0.4} \mathrm{Ga}_{0.6}$ As quantum wells with (solid curve) and without (dashed curve) strained $\mathrm{In}_{0.2} \mathrm{Ga}_{0.8} A$ As guarding layers samples. Note that the intensity is in log scale.

samples is about 17. For sample sets 2 and 3, the PL intensity ratios for the QW peaks are 5 and 3, respectively. These results strongly suggest that the bottom strained $\mathrm{In}_{0.2} \mathrm{Ga}_{0.8}$ As layer can prevent defects originating in the substrate from propagating into the GaAs QW.

After the implantation-RTA treatments, significant changes in the PL intensities were observed. Figure 4 shows the results for set 1 , in which the PL peak intensity of the QW emission in structure $B$ is twice that in the as-grown sample while the PL intensity of structure A decreased by a factor of 4 , compared to the as-grown sample. The PL intensity ratio of the QW peaks in the two samples is therefore as high as 130 . These changes are due to the combined effects of RTA and silicon implantation. RTA itself may reduce the density of defects. In fact, we have observed the enhancement of PL intensity in several four-QW strained AlGaAs/GaAs/InGaAs gradedrefractive-index separate-confinement-heterostructure (GRIN-SCH) lasers after RTA. After annealing, the PL intensities from the wells can increase 5-10 times. Lasers of broad-area geometry made from these samples showed that, after RTA treatment, the reduction in threshold current density can be as high as sevenfold. This improvement might be due to the reduced defect densities at heterointerfaces in the active region. In the present study, a high density of defects was produced in the top $\mathrm{Al}_{0.4} \mathrm{Ga}_{0.6}$ As layer by implantation. Most of these defects were annealed after RTA. However, some residual defects may remain and propagate in the structure during the thermal cycle. Since structure $B$ has a pseudomorphic $\operatorname{In}_{0.2} \mathrm{Ga}_{0.8}$ As layers, the QW, instead of degrading due to defect propagation into it, benefits from the reduction of defects by RTA. This results in the increased PL intensity in structure $B$. On the other hand, structure A, which has no strained layers, suffers from the propagation of a large density of defects into the $\mathrm{QW}$ region, resulting in reduced $\mathrm{PL}$ intensity. The annealed samples also exhibited significant enhancement in the intensities of the PL signal from the substrate after annealing.

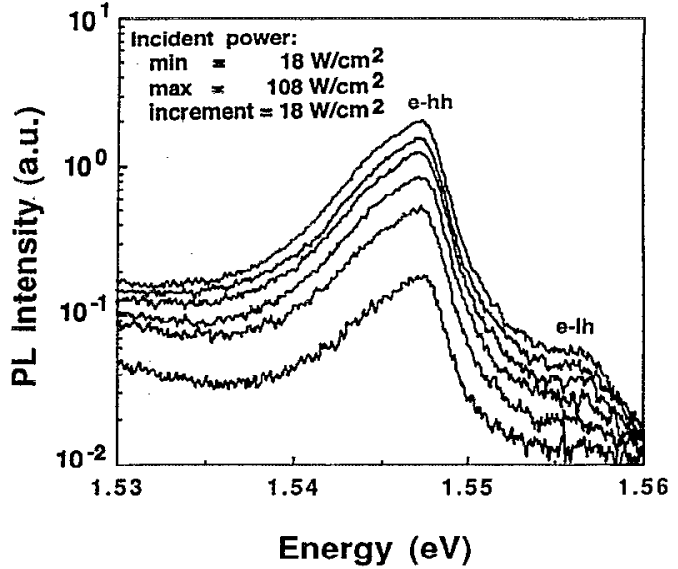

FIG. 5. Intensity variation of the heavy-hole and light-hole peaks of $Q W$ photoluminescence with increasing incident laser power. Note that the intensity is in log scale.

One may wonder if the surface remained the same before and after RTA since a rough surface would result in smaller reflectivity. If surface roughness is indeed playing a significant role in our experiment, we would expect the annealed samples to have rougher surfaces and, thus, lower PL intensities. However, we found increased PL intensities in structure B after annealing. Therefore, the possible effect of surface roughness due to RTA can be ruled out.

One may notice that the PL line shapes of structures A and $\mathbf{B}$ are different. In the PI, spectra of samples with structure $B$, there is a small peak with energy about 10 meV higher than that of the major QW peak. The PL intensities of these peaks increase linearly with incident laser power. Figure 5 shows the variation of the PL intensity of these two peaks with incident laser power for structure $B$ in set 1 after the implantation-RTA process. The incident power varied from 18 to $108 \mathrm{~W} / \mathrm{cm}^{2}$ with an increment of $18 \mathrm{~W} / \mathrm{cm}^{2}$. At higher incident powers, the weak peak becomes stronger. We believe that this peak is due to electron-to-light-hole ( $e$-lh) transitions while the strong main peak is due to electron to heavy-hole (e-hh) transitions. The light-hole peak was seen only in samples with structure B, both before and after the implantation-RTA process, but not in any sample of structure $A$. This difference again suggests that structure $B$ has a $Q W$ of superior optical quality.

The superior optical quality of quantum wells in structures having the pseudomorphic barrier layers has important implications in MBE growth of semiconductor heterostructures and devices. It is a standard practice to deposit a smoothing buffer layer of thickness 3000-5000 $\AA$ before the growth of the active region. This study suggests that the thickness of the smoothing layer can be greatly reduced by using a pseudomorphic layer. The growth time thus can be reduced and a lot of resources can be saved. In addition, impurity diffusion problems may also be minimized in devices. This aspect is being currently investigated. 


\section{CONCLUSIONS}

In summary, we have shown with the help of a simple model that, in a semiconductor heterostructure, defects originating from lattice-matched regions can be prevented from entering, or can be trapped by, a pseudomorphic layer, depending on the signs of the strain in the pseudomorphic layer and the strain induced by the defect. A pseudomorphic layer can thus be used to prevent defect propagation into the critical active region of a structure. Experimentally, we have compared the PL intensities of $\mathrm{Al}_{0.4} \mathrm{Ga}_{0.6} \mathrm{As} / \mathrm{GaAs} \mathrm{QWs}$ with and without pseudomorphic $\mathrm{In}_{0.2} \mathrm{Ga}_{0.8}$ As layers for prevention of defect propagation. Defect propagation was induced intentionally by the use of high-EPD substrates, by implantation as well as RTA. The structures with $\mathrm{In}_{0.2} \mathrm{Ga}_{0.8}$ As layers showed much higher QW PL intensities (about 130 times) than those without the pseudomorphic layers. These results strongly support the model of defect propagation.

\section{ACKNOWLEDGMENT}

This work is supported by the Office of Naval Research under Grant No. N00019-89-J1519.

${ }^{\prime}$ G. I. Ng, W.-P. Hong, D. Pavlidis, M. Tutt, and P. K. Bhattacharya, Electron. Dev. Lett. 9, 439 (1988).

${ }^{2}$ T. J. Drummond, T. E. Zipperian, I. J. Fritz, J. E. Schirber, and T. A. Plut, Appl. Phys. Lett. 49, 461 (1986).

${ }^{3}$ A. R. Adams, Electron. Lett. 22, 249 (1986).

${ }^{4}$ E. Yablonovitch and E. O. Kane, IEEE J. Lightwave Technol. LT-5, 504 (1986).

${ }^{5}$ S. W. Corzine and L. A. Coldren, Appl. Phys. Lett. 59, 588 (1991).

${ }^{6}$ H. K. Choi, C. A. Wang, D. F. Kolesar, R. L. Aggarwal, and J. N. Walpole, IEEE Photon. Technol. Lett. PTL-3, 857 (1991).

${ }^{7}$ S. L. Yellen, R. G. Waters, A. H. Shepard, J. A. Baumann, and R. J. Dalby, IEEE Photon. Technol. Lett. PTL-4, 829 (1992).

${ }^{8} J$. M. Matthews and A. E. Blakeslee, J. Cryst. Growth 27, 118 (1974). ${ }^{9}$ See, for example, A. P. Boresi, Elasticity in Engineering Mechanics (Prentice-Hall, Englewood Cliffs, NJ, 1965), Chap. 4. 